\title{
High LIN28A and PLK4 co-expression is associated with poor prognosis in epithelial ovarian cancer
}

\author{
YAO HE ${ }^{1-3^{*}}$, HUI WANG ${ }^{1,4^{*}}$, MEINA YAN $^{1,4^{*}}$, XINXIN YANG ${ }^{1,4}$, RONG SHEN $^{1,4}$, \\ XIAOGE NI ${ }^{5}$, XIAOKUN CHEN ${ }^{2}$, PEIFANG YANG ${ }^{2}$, MIAO CHEN $^{1}$, XIAODONG LU $^{1}$, \\ GENBAO SHAO $^{1}$, XIAOMING ZHOU ${ }^{1}$ and QIXIANG SHAO ${ }^{1,4}$
}

\author{
${ }^{1}$ Department of Immunology, School of Medicine, Jiangsu University, Zhenjiang, Jiangsu 212013; \\ ${ }^{2}$ Department of Gynecology and Obstetrics, Affiliated Hospital of Jiangsu University, Zhenjiang, Jiangsu 212001; \\ ${ }^{3}$ Department of Obstetrics, Obstetrics and Gynecology Hospital Affiliated to Nanjing Medical University, Nanjing, \\ Jiangsu 2100011; ${ }^{4}$ Jiangsu Key Laboratory of Medical Science and Laboratory Medicine, Zhenjiang, Jiangsu 212013; \\ ${ }^{5}$ Department of Gynecology and Obstetrics, Affiliated People's Hospital of Jiangsu University, \\ Zhenjiang, Jiangsu 212001, P.R. China
}

Received October 31, 2017; Accepted July 30, 2018

DOI: $10.3892 / \mathrm{mmr} .2018 .9562$

\begin{abstract}
Epithelial ovarian cancer (EOC) is the most lethal gynecological malignancy. LIN28 homolog A (LIN28A) is a RNA-binding protein, which serves a fundamental role in cell development and pluripotency. Polo-like kinase 4 (PLK4) is a member of the polo-like kinase family, which primarily takes part in the mitotic regulation. Overexpression of LIN28A has been demonstrated in ovarian cancer; however, the expression of PLK4 and the correlation between the expression of LIN28A and PLK4 in EOC has not been discussed. In the present study, the mRNA and protein levels of LIN28A and PLK4 were evaluated by reverse transcription-quantitative polymerase chain reaction and immunohistochemistry in ovarian tissues of patients. Results demonstrated significantly increased expression in EOC compared with benign epithelial ovarian tumors. High expression of LIN28A and PLK4 was detected at the advanced pathological stage. Furthermore, PLK4 expression was positively correlated with LIN28A $(\mathrm{r}=0.555 ; \mathrm{P}=0.039)$. The median survival analysis of patients with EOC with LIN28A and PLK4 double positive expression was 14 months, compared with 30 months in single positive and 60 months in double negative patients by Kaplan-Meier analysis $(\mathrm{P}<0.05)$. The expressions of LIN28A and PLK4 was elevated in different EOC cell lines compared to with a normal
\end{abstract}

Correspondence to: Professor Qixiang Shao, Department of Immunology, School of Medicine, Jiangsu University, 301 Xuefu Road, Zhenjiang, Jiangsu 212013, P.R. China

E-mail: shao_qx@ujs.edu.cn

*Contributed equally

Key words: epithelial ovarian cancer, LIN28 homolog A, polo-like kinase 4, prognosis, co-expression ovarian cell line. The 293 T cells transfected with LIN28A plus a PLK4 plasmid were the fastest-growing group. These results suggest that co-expression of LIN28A and PLK4 may be associated with poor prognosis of EOC and could serve as promising prognostic biomarkers and therapeutic targets in EOC. LIN28A and PLK4 may be used along with traditional morphological and clinical characteristics for predicting prognosis.

\section{Introduction}

Ovarian cancer incidence ranks second among gynecological cancers in women; while the mortality rate is the highest, accounting for $>151,000$ mortalities annually (1). Due to the anatomical location of the ovaries, deep within the pelvis, no obvious clinical symptoms are observed in the early stage of the disease (2); thus, 70-80\% of women have reached an advanced stage by the time of diagnosis, with peritoneal diffusion or distant metastasis. This brings great challenges for the treatment of ovarian cancer (3). Ovarian cancer can be categorized into epithelial and non-epithelial groups. Epithelial ovarian cancer (EOC) accounts for $85-90 \%$ of ovarian cancers (4). Despite the initial therapeutic strategy of cytoreductive surgery combined with platinum or taxane chemotherapy, $>60 \%$ of patients still experience relapse and resistance. Platinum sensitivity is considered to be an important factor of prognosis. Of the patients with advanced ovarian cancer, $\sim 80 \%$ have recurrence within 3 years and die within 5 years (5). Therefore, a better understanding regarding the mechanism of EOC progression, reliable prognostic markers, therapeutic targets and combination therapies are required.

Cancer stem cells (CSCs), which typically accounts for $0.01-1.0 \%$, are reported to be the main source of the recurrence of EOC (6). Therefore, the use of CSC markers is proposed for prognostic diagnosis. In previous years, LIN28 homolog A (LIN28) protein has increasingly been considered as a special kind of stem cell factor that blocks the correct 
post-transcriptional processing of the let-7 microRNA (miR) family and mRNAs involved in cell growth and metabolism to maintain stem cell activity $(7,8)$. LIN28 is a highly conserved RNA-binding protein that is currently only established to be present in eukaryotic cells, and there are two homologues, LIN28A and LIN28B. The LIN28 protein homolog has two common RNA binding domains, a pair of retroviral type of CCHC zinc finger proteins and a cold shock region (9). LIN28 protein is mainly expressed in the cytoplasm and under different metabolic pressures it is expressed in embryonic cancer cells and myoblasts. LIN28 is localized in the nucleus, when the RNA-binding region is mutated (10). High LIN28A levels are correlated with advanced human malignancies. LIN28 can influence the development of tumors by direct or synergistic effects on the expression of various oncogenes, including erb-b2 receptor tyrosine kinase 2 (HER2) and high mobility group AT-hook 1 (HMGA1) in breast cancer (11). In ovarian cancer, LIN28 can increase the growth of ovarian cancer cells by promoting the expression of bone morphogenetic proteins and POU class 5 homeobox 1 (Oct4). The co-expression of LIN28 and Oct4 frequently suggests poor prognosis in ovarian cancer $(12,13)$. Our previous microarray data has suggested that the expression of LIN28A was significantly increased in A2780 cells compared with the normal human ovarian epithelial cell line, HOSEPIC (unpublished data).

Polo-like kinases (PLKs) are a family of serine-threonine kinases that are involved in various cell cycle-associated processes, including DNA replication, mitosis, and centrosome maturation. Currently, five members of the PLK family have been identified, and they contain a highly conserved $\mathrm{N}$-terminal kinase domain and C-terminal polo box domain (14). Abnormal expression of PLKs as been observed in multiple types of cancer. It is well established that PLK1 is increased in a broad range of cancer tissues, including colorectal cancer, hepatocellular carcinoma, non-small cell lung cancer and gastric adenocarcinoma (15). PLK1 overexpression is correlated with the progression of several types of cancers, and PLK1 inhibitors have been tested in clinical trials (16). In an early study, overexpression of PLK1 in ovarian cancer was associated with high-grade cancer. PLK4 has a key role in centriole replication, while PLK4 inhibition has been considered as a potential approach to treat chromosomally unstable cancer including prostate cancer, by disrupting mitosis (17). High PLK4 expression was also detected in certain gynecological tumors. The levels of PLK4 mRNA was significantly higher in breast cancer tissues compared with normal breast tissues (18). High PLK4 expression is associated with poorer prognosis and increased resistance to taxane-based neoadjuvant chemotherapy (19). However, little is known about the importance of PLK4 in ovarian cancer. Whether PLK4 has the potential to be a prognostic factor in ovarian cancer and a predictor of response to chemotherapy needs to be examined. Both LIN28A and PLK4 can regulate a dual specificity phosphatase, cell division cycle 25 (Cdc25), which has three isoforms, Cdc25A, Cdc25B and $\mathrm{Cdc} 25 \mathrm{C}$. Cdc25 is a key mediator in driving the cell cycle by activating cyclin dependent kinase complexes. It also acts as an effector of DNA damage checkpoints. LIN28A can regulate the expression level of Cdc25A, which is dependent on let-7 (13). PLK4 phosphorylates Cdc25C, resulting in translocation to the nucleus, triggering mitosis (20).
To the best of our knowledge, no study has examined the expression of PLK4 in ovarian cancer. Therefore, in the present study, the expression patterns of LIN28A and PLK4 were investigated in ovarian cancer tissues and cell lines using reverse transcription-quantitative polymerase chain reaction (RT-qPCR), western blotting and immunohistochemistry. Furthermore, the correlation between LIN28A and PLK4, and their association with clinical features, were examined.

\section{Materials and methods}

Human tissue specimen. A total of 79 paraffin-embedded tissue samples (age range, 23-77), including 31 benign ovarian samples and 48 EOC samples, were collected from the Affiliated Hospital of Jiangsu University (Zhenjiang, China) and the Affiliated People's Hospital of Jiangsu University (Zhenjiang, China) between July 2009 and July 2014. The benign ovarian tissues were obtained from 31 patients with uterine fibroids, abdominal masses and other diseases, including ovarian removal or resection surgery, but not with uterine or cervical and other malignant tumors. The age of the patients ranged from 22 to 77 years with a median age of 53 years. All 48 EOC samples were obtained from the patients with primary EOC that received no anti-tumor therapy prior to surgery and had completely removed the ipsilateral ovaries. The patient's age ranged from 23-70 years with a median age of 45 years in this group. The classification of cancer stage was done according to the International Federation of Gynecology and Obstetrics (FIGO; 2009) (21). The specimens included 15 cases of stage I, 12 cases of stage II, 20 cases of stage III and 1 case of stage IV. Histological type was confirmed by microscopic examination of the hematoxylin and eosin-stained slides. The study was approved by the Ethics Committee of Jiangsu University and informed consent was obtained from all the recruited subjects.

Immunohistochemistry (IHC) assays. For IHC, paraffin-embedded sections $(5-\mu \mathrm{m}$ in thickness) were deparaffinized in graded alcohol (70-100\%), and 100\% xylene at room temperature. The sections were then immersed in $10 \mathrm{mM}$ sodium citrate buffer and antigens were retrieved by microwaving for $30 \mathrm{~min}$. Sections were quenched in $3 \% \mathrm{H}_{2} \mathrm{O}_{2}$ for $15 \mathrm{~min}$ and blocked with $5 \%$ normal goat serum (OriGene Technologies, Inc., Beijing, China) at room temperature for $1 \mathrm{~h}$. The sections were incubated overnight at $4^{\circ} \mathrm{C}$ with the primary antibodies diluted in 1:100 ratio. The sections were then incubated with HRP-conjugated goat anti-rabbit IgG secondary antibody (cat. no. TA140003; OriGene Technologies, Inc.) at room temperature for $1 \mathrm{~h}$. Then, 5\% 3,3'-diaminobenzidine tetrahydrochloride solution (OriGene Technologies, Inc.) was added for 1-3 min at room temperature. The nuclei were counterstained with $1 \%$ hematoxylin for $3 \mathrm{~min}$ at room temperature. The sections were dehydrated and covered with a coverslip using permount. The images were captured with a light microscope (DP70, Olympus Corporation, Tokyo, Japan). The IHC staining score was graded according to the staining intensity and area extent and evaluated by two pathologists who were blinded to the clinicopathological variables. A semiquantitative assessment was used to describe the percentage of positive stained cells. The IHC staining was scored as 
negative (no cells stained) and positive $(+,<25 \%$ positive cells; ,$++ 25-50 \%$ positive cells;,$+++>50 \%$ positive cells).

Cell lines. The human ovarian surface epithelial cell line (HOSEPIC) were provided by from Professor Genbao Shao, OVCAR-3 from Professor Xiaoming Zhou, SKOV3, 3AO, A2780 and HO8910 from Professor Xiaodong Lu and 293T cells from Professor Wenrong Xu (all Jiangsu University, Zhenjiang, China). Cells were maintained in Dulbecco's modified Eagle's medium (Gibco; Thermo Fisher Scientific, Inc. Waltham, MA, USA) supplemented with $10 \%$ fetal bovine serum (Gibco; Thermo Fisher Scientific, Inc.) at $37^{\circ} \mathrm{C}$ in a humidified $5 \% \mathrm{CO}_{2}$ atmosphere. Plasmids pSin-EF2-Lin28-Pur and pWZL-Neo-Myr-Flag-PLK4 were purchased from Addgene, Inc. (Cambridge, MA, USA). Plasmids were transfected using Lipofectamine ${ }^{\circledR} 2000$ (Thermo Fisher Scientific, Inc.) according to the manufacturer's protocol. Briefly, 293T cells were seeded at $3 \times 10^{5}$ per well into 6 -well plate and were transfected with $2 \mu \mathrm{g}$ pcDNA3.1 empty vector, LIN28A plasmid, PLK4 plasmid independently or co-transfected with $2 \mu \mathrm{g}$ LIN28A plus PLK4 plasmids, respectively. Cells were harvested 24 and $48 \mathrm{~h}$ post-transfection for the evaluation of mRNA and protein expression. A hemocytometer was used to count total cell number.

$R T-q P C R$. Total RNA was extracted using TRIzol reagent (Takara, Bio Inc., Otsu, Japan; cat. no. 9109). RNA (1 $\mu \mathrm{g})$ was reverse transcribed into cDNA with PrimeScript ${ }^{\mathrm{TM}}$ RT Reagent kit (Takara Bio Inc.; cat. no. RR037A; $37^{\circ} \mathrm{C}$ for $15 \mathrm{~min}, 85^{\circ} \mathrm{C}$ for $5 \mathrm{sec}$ ). Human $\beta$-actin RNA was used as an internal control. Primers for LIN28A and PLK4 are as follows: LIN28A forward, 5'-AGGCGGTGGAGTTCACCT TTAAGA-3' and reverse 5'-AGCTTGCATTCCTTGGCA TGATGG-3'; PLK4 forward, 5'-AATCAAGCACTCTCC AATC-3' and reverse, 5'-TGTGTCCTTCTGCAAATC-3'; $\beta$-actin forward, 5'-GTTGCGTTACACCCTTTCTTG-3' and reverse, 5'-CACCTTCACCGTTCCAGTTT-3'. Gene expression levels were evaluated by qPCR with SYBR Premix (Takara Bio, Inc.; cat. no. RR820A), using the $2^{-\Delta \Delta C q}$ method (22).

Reagents and antibodies. The specific primary antibodies used in this study were anti-PLK4 antibody (Abcam, Cambridge, UK; cat. no. ab137398), rabbit anti-LIN28A antibody (cat. no. 8641; Cell Signaling Technology, Inc., Danvers, MA, USA), rabbit anti-Ki67 antibody (cat. no. ab15580; Abcam) and mouse anti- $\beta$-actin antibody (cat. no. sc47778; Santa Cruz Biotechnology, Inc., Dallas, TX, USA). Secondary antibodies used were horseradish peroxidase (HRP)-conjugated anti-rabbit IgG or anti-mouse IgG (cat. nos. 7074 and 7076, respectively; Cell Signaling Technology, Inc.) at a 1:10,000. Cell protein extraction reagent was obtained from CWBioTec (cat. no. CW0889; Jiangsu Kangwei Century Biotechnology Co., Ltd., Beijing, China).

Western blot analysis. Confluent cells were lysed using lysis buffer (cat. no. CW0889; 0.5\% $\mathrm{C}_{24} \mathrm{H}_{40} \mathrm{O}_{4} \cdot \mathrm{Na}, 10 \mathrm{mM}$ Tris- $\mathrm{HCl}$ pH 7.8, 0.5\% Nonidet P-40, $10 \mathrm{mM}$ EDTA and $100 \mathrm{mM} \mathrm{NaCl}$ ) in the presence of protease inhibitor cocktail and protein concentration was determined using a bicinchoninic acid reagent (Merck KGaA, Darmstadt, Germany). Aliquots of
$10 \mu 1$ protein samples were separated on 10 or $12 \%$ SDS-PAGE and electronically transferred onto polyvinlylidene difluoride membranes. The samples were blocked with $5 \%$ bovine serum albumin (Shanghai Yeasen Biotechnology Co., Ltd, Shanghai, China) at room temperature for $30 \mathrm{~min}$, then probed using various primary antibodies with a dilution of $1: 1,000$ at $4{ }^{\circ} \mathrm{C}$ overnight. The samples were then washed with Tris-buffered saline at $\mathrm{pH} 7.6$ containing TBS Tween-20 (1\%) three times. These were further incubated with HRP-conjugated secondary antibodies at a dilution of 1:10,000 for $2 \mathrm{~h}$ at room temperature. Blots were developed using an Enhanced Chemo Luminescence system (ECL; EMD Millipore, Billerica, MA, USA) and visualized using ImageQuant LAS 400 mini ECL system (GE Healthcare, Chicago, IL, USA). Densitometric analysis was performed with Image Pro Plus software version 6.0 (Media Cybernetics, Inc., Rockville, MD, USA).

Statistical analysis. SPSS software version 17.0 (SPSS, Inc., Chicago, IL, USA) was used for statistical analysis. Results from at least three separate experiments were expressed as the mean \pm standard deviation. Statistical differences between multiple groups were assessed by one-way analysis of variance followed by Tukey's post-hoc test. A t-test was used for comparison between two groups. Differences between the groups were estimated using the $\chi^{2}$ test. Spearman's rank correlation test was used to analyze the association between the two proteins. Kaplan-Meier curves were utilized to assess patient survival period, and statistical significance in the survival period between the patient groups were analyzed using log-rank test. Cox's proportional hazards model was used for univariate and multivariate analysis. $\mathrm{P}<0.05$ was considered to indicate a statistically significant difference.

\section{Results}

High expression of LIN28A and PLK4 in human EOC. RT-qPCR was first used to analyze the mRNA levels of LIN28A and PLK4 in the EOC tissues, and compared the levels with benign ovarian tissues. The mRNA levels of LIN28A in the EOC group were significantly increased compared with the benign group $(\mathrm{P}<0.05)$, and similar results were observed for PLK4 ( $\mathrm{P}<0.01$; Fig. 1). The protein expression of LIN28A and PLK4 in different ovarian tissues was further studied using IHC staining (Fig. 2). Results identified no expression of LIN28A protein and PLK4 in the benign ovarian tissues. However, positive expression of LIN28A and PLK4 was demonstrated in 9 of 48 cases $(18.8 \%)$ and 13 of 49 cases $(27.1 \%)$, respectively, in the EOC tissues. Pearson's $\chi^{2}$ test revealed that the EOC group had increased expression of LIN28A and PLK4 compared with the benign ovarian tissues $(\mathrm{P}<0.05$; Table I). Therefore, these data suggest increased expression of LIN28A and PLK4 in human EOC specimens.

Association of LIN28A and PLK4 with clinicalpathological parameters. The clinicopathological features of EOC, including patient age, pathological stage and histological type, were collected and then the association of LIN28A and PLK4 levels with these clinical parameters was determined. The expression of LIN28A and PLK4 was not associated with age and tissue type $(\mathrm{P}>0.05)$; whereas LIN28A and 
A

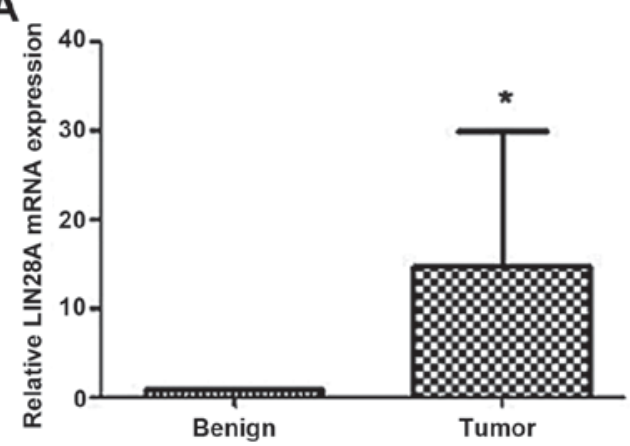

B

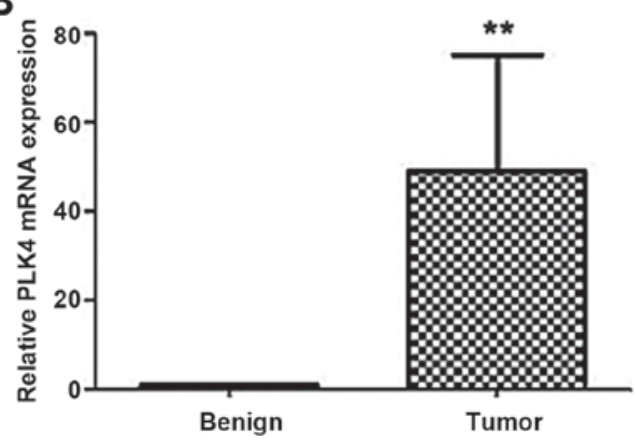

Figure 1. Comparative analyses of mRNA levels of LIN28A and PLK4 in different ovarian tissues. (A) mRNA levels of LIN28A were detected by RT-qPCR in benign epithelial ovarian tissues and epithelial ovarian cancer. (B) mRNA levels of PLK4 were detected by RT-qPCR in benign epithelial ovarian tissues and epithelial ovarian cancer. Data are presented as the mean \pm standard deviation. ${ }^{*} \mathrm{P}<0.05$ and ${ }^{* *} \mathrm{P}<0.01$ vs. benign. RT-qPCR, reverse transcription-quantitative polymerase chain reaction; LIN28A, LIN28 homolog A; PLK4, Polo-like kinase 4.

A

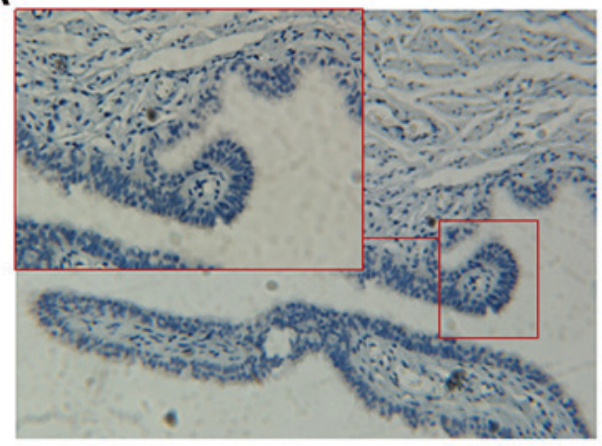

C

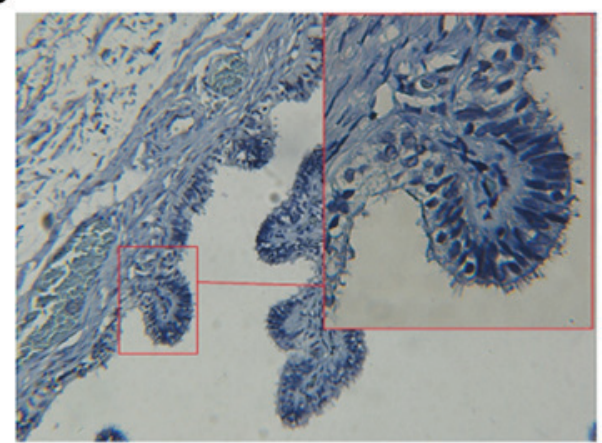

B

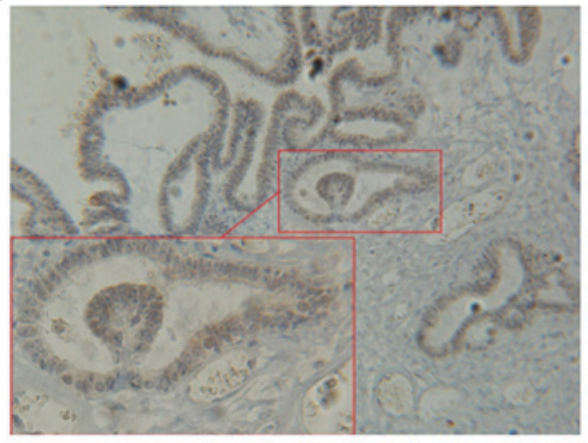

D

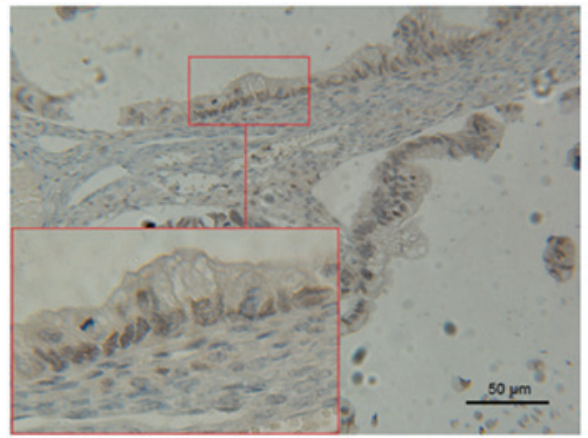

Figure 2. Immunohistochemistry analyses of LIN28A and PLK4 expression in different ovarian tissues. (A) Expression of LIN28A in benign epithelial ovarian tissues. (B) Expression of LIN28A in epithelial ovarian cancer. (C) Expression of PLK4 in benign epithelial ovarian tissues. (D) Expression of PLK4 in epithelial ovarian cancer. Micrographs were captured using a Zeiss microscope. The corner (magnification, x400) was an enlargement of every micrograph (magnification, x200). LIN28A, LIN28 homolog A; PLK4, Polo-like kinase 4.

PLK4-positivity was significantly associated with pathological stage (FIGO staging; $\mathrm{P}<0.05$; Table II). The cancer specimens included 15 cases at stage I, 12 at stage II, $20 \mathrm{t}$ stage III and 1 at stage IV. Histological type was confirmed by microscopic examination of hematoxylin and eosin-stained slides, and demonstrated that 37 cases were serous carcinoma and 11 cases were mucinous carcinoma (Table II). These results suggest that the expression of LIN28A and PLK4 was significantly increased at stages III/IV compared with I/II.

Correlation between LIN28A and PLK4 in EOC. Since both LIN28A and PLK4 can regulate G2/M cell cycle substrate $\mathrm{Cdc} 25$, and are positively expressed in EOC, the Spearman's rank correlation test was used to analyze the association between the two proteins. IHC results demonstrated that the expression of the PLK4 protein was positively correlated with the expression of LIN28A protein ( $\mathrm{r}=0.555, \mathrm{P}=0.039$; data not shown). This suggested increased expression of PLK4 protein was correlated with the increased expression level of the LIN28A protein.

Correlation of LIN28A and PLK4 co-expression with poor prognosis. A total of $48 \mathrm{EOC}$ patients were followed up for 7-70 months, and the median follow-up period was 33 months. At the end of the follow-up period, a total of 30 patients survived and 18 patients died. The survival time of patients 
Table I. LIN28A and PLK4 expression in different ovarian tissues.

\begin{tabular}{|c|c|c|c|c|c|c|c|c|c|}
\hline \multirow[b]{2}{*}{ Type } & \multicolumn{5}{|c|}{ LIN28A } & \multicolumn{4}{|c|}{ PLK4 } \\
\hline & Cases & Positive & Negative & Positive rate $(\%)$ & $\mathrm{P}$ & Positive & Negative & Positive rate $(\%)$ & $\mathrm{P}$ \\
\hline Benign & 31 & 0 & 31 & 0 & & 0 & 31 & 0 & \\
\hline Tumor & 48 & 9 & 39 & 18.8 & 0.027 & 13 & 35 & 27.1 & 0.01 \\
\hline
\end{tabular}

PLK4, Polo-like kinase 4; LIN28A, LIN28 homolog A.

Table II. Association between LIN28A and PLK4 expression and clinical variables.

\begin{tabular}{|c|c|c|c|c|c|}
\hline \multirow[b]{2}{*}{ Parameters } & \multirow[b]{2}{*}{ Cases } & \multicolumn{2}{|c|}{ LIN28A } & \multicolumn{2}{|c|}{ PLK4 } \\
\hline & & Positive (\%) & P-value & Positive (\%) & P-value \\
\hline Age, years & & & 0.885 & & 0.788 \\
\hline$<50$ & 17 & $3(17.6)$ & & $5(29.4)$ & \\
\hline$\geq 50$ & 31 & $6(19.4)$ & & $8(25.8)$ & \\
\hline Pathological stage & & & 0.022 & & 0.03 \\
\hline $\mathrm{I} / \mathrm{II}$ & 27 & $2(7.4)$ & & $4(14.8)$ & \\
\hline III/IV & 21 & $7(33.3)$ & & $9(42.9)$ & \\
\hline Histology & & & 0.906 & & 0.499 \\
\hline Serous & 37 & 7 (18.9) & & $11(29.7)$ & \\
\hline Mucinous & 11 & $2(18.2)$ & & $2(18.2)$ & \\
\hline
\end{tabular}

PLK4, Polo-like kinase 4; LIN28A, LIN28 homolog A.

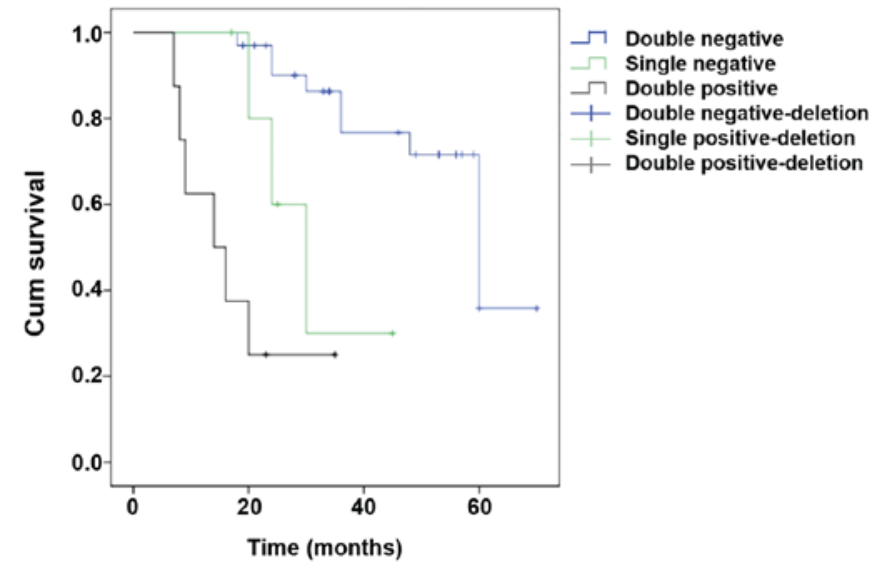

Figure 3. Kaplan-Meier survival curves for LIN28A and PLK4 expression in epithelial ovarian cancer. The overall survival of patients with LIN28A-negative and PLK4-negative (double negative), LIN28A-positive or PLK4-positive (single positive), and PLK4-positive and LIN28A-positive (double positive) expression was plotted. The survival time is known to be longer than the observed deleted time, since the survival includes discontinued follow-up and mortality by other causes. Deleted indicates that the patients' survival time is unknown. Cum, cumulative; PLK4, Polo-like kinase 4; LIN28A, LIN28 homolog A.

co-expressing LIN28A and PLK4 was from 7-23 months, and the median time was 14 months. The survival time of patients with LIN28A-positive or PLK4-positive tumors was from 20-45 months, and the median time was 30 months. The survival time of patients with LIN28A and PLK4-negative tumors was 18-70 months, and the median time was 60 months. The survival of double positive patients was significantly shorter than the double negative patients $\left(\chi^{2}=29.62463\right.$; $\mathrm{P}<0.001$; Fig. 3).

A single factor logistic regression analysis was performed to assess the prognosis factors of patients with EOC. Results demonstrated that LIN28A [odds ratio $(\mathrm{OR})=11.667$, $\mathrm{P}=0.004,95 \%$ confidence interval $(\mathrm{CI})=2.050-66.408)]$, PLK4 (OR=5.400, $\mathrm{P}=0.018,95 \% \mathrm{CI}=1.375-21.205)$ and pathological stage $(\mathrm{OR}=10.000, \mathrm{P}=0.026,95 \% \mathrm{CI}=0.972-102.868)$ were considered as significant prognostic factors for EOC. Multivariate Cox regression analysis demonstrated that there was no independent risk factor for the prognosis of patients with EOC. However, co-expression of LIN28A and PLK4 indicates poor prognosis (Table III).

Expression of LIN28A and PLK4 in ovarian cell lines. To confirm the role of LIN28A and PLK4 in EOC, the expression of LIN28A and PLK4 were determined in normal human ovarian epithelial cells, HOSEPIC, and other human EOC cell lines, including 3AO, A2780, HO8910, OVCAR-3 and SKOV3 via RT-qPCR. The results of RT-qPCR demonstrated that the expression level of LIN28A in A2780 and HO8910 was significantly increased compared with the HOSEPIC cell line $(\mathrm{P}<0.001)$, and the expression level of LIN28A was highest in the A2780 cell line, which may be associated with the growth rate (Fig. 4A and $\mathrm{B}$ ). Compared with HOSEPIC, LIN28A 
Table III. Univariate and multivariate analysis of variables with overall survival.

\begin{tabular}{|c|c|c|c|c|c|c|}
\hline \multirow[b]{2}{*}{ Variable } & \multicolumn{3}{|c|}{ Univariate analysis } & \multicolumn{3}{|c|}{ Multivariate analysis } \\
\hline & OR & $95 \% \mathrm{CI}$ & P-value & OR & $95 \% \mathrm{CI}$ & P-value \\
\hline LIN28A & 11.667 & $2.050-66.408$ & 0.004 & 18.530 & $0.788-435.872$ & 0.070 \\
\hline PLK4 & 5.400 & $1.375-21.205$ & 0.018 & 3.491 & $0.319-38.260$ & 0.306 \\
\hline Pathological stage & 10.000 & $0.972-102.868$ & 0.026 & 24.086 & $1.442-402.253$ & 0.085 \\
\hline Histology & 1.970 & $0.495-7.832$ & 0.468 & 3.301 & $0.493-22.085$ & 0.218 \\
\hline Age & 3.370 & $0.801-14.177$ & 0.116 & 4.095 & $0.519-32.340$ & 0.181 \\
\hline Ki67 & 0.417 & $0.042-4.085$ & 0.581 & 0.400 & $0.021-7.760$ & 0.832 \\
\hline
\end{tabular}

PLK4 expression was determined by immunohistochemical analysis. OR, odds ratio; CI, confidence interval; PLK4, Polo-like kinase 4; LIN28A, LIN28 homolog A.
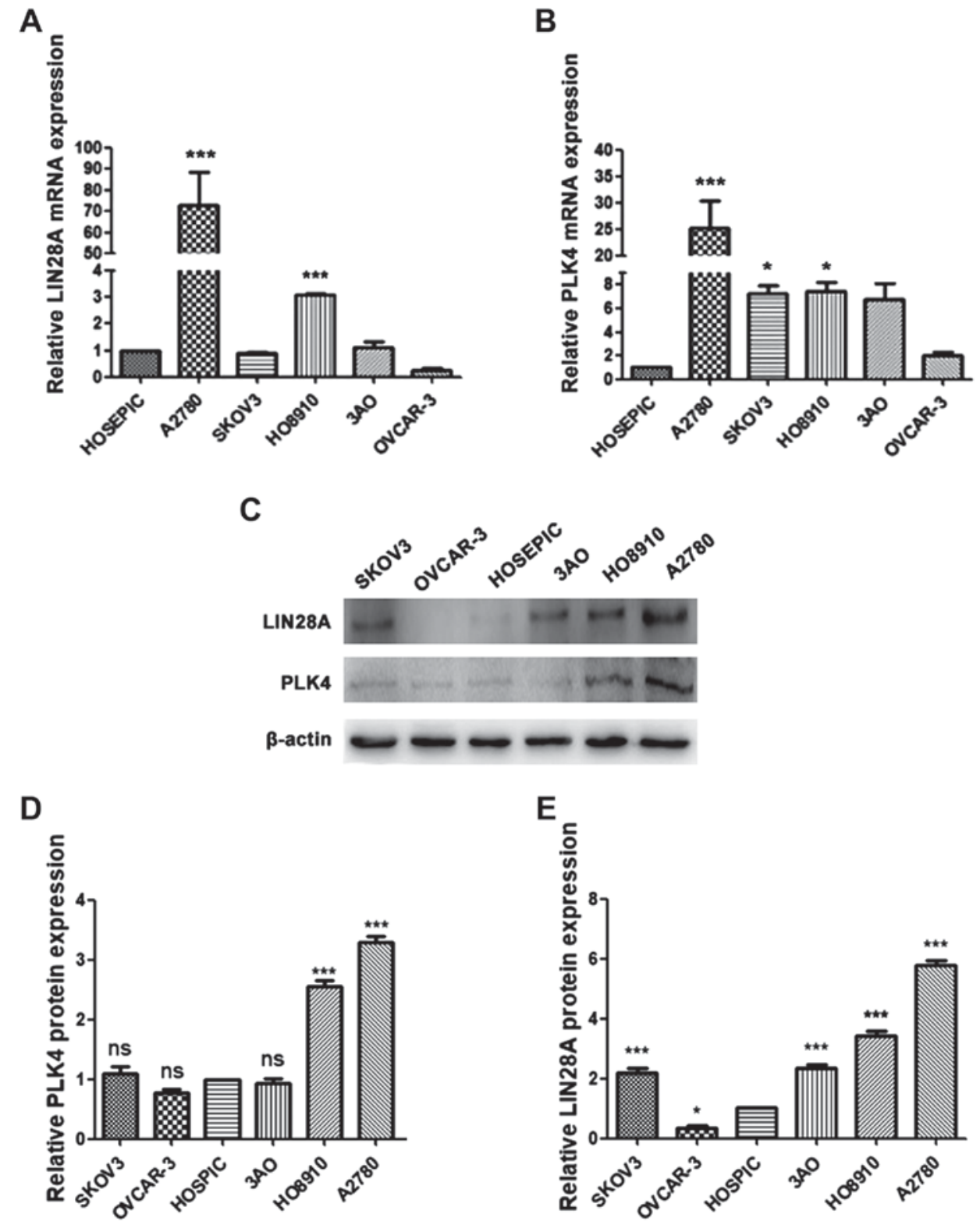

Figure 4. mRNA and protein levels of LIN28A and PLK4 in different ovarian cell lines. Normal epithelial ovarian cells, HOSEPIC, were used as a control. A2780, SKOV3, HO8910, 3AO and OVCAR-3 were epithelial ovarian cancer cell lines. The mRNA levels of (A) LIN28A and (B) PLK4 were detected with RT-qPCR in these cell lines. (C) Western blotting of LIN28A and PLK4 in different ovarian cell lines. Quantitative analyses of each gray numerical value of (D) PLK4 and (E) LIN28A vs. $\beta$-actin. Statistical differences were compared with HOSEPIC. ${ }^{*} \mathrm{P}<0.05,{ }^{* *} \mathrm{P}<0.001$ vs. HOSEPIC. RT-qPCR, reverse transcription-quantitative polymerase chain reaction; PLK4, Polo-like kinase 4; LIN28A, LIN28 homolog A. 
A
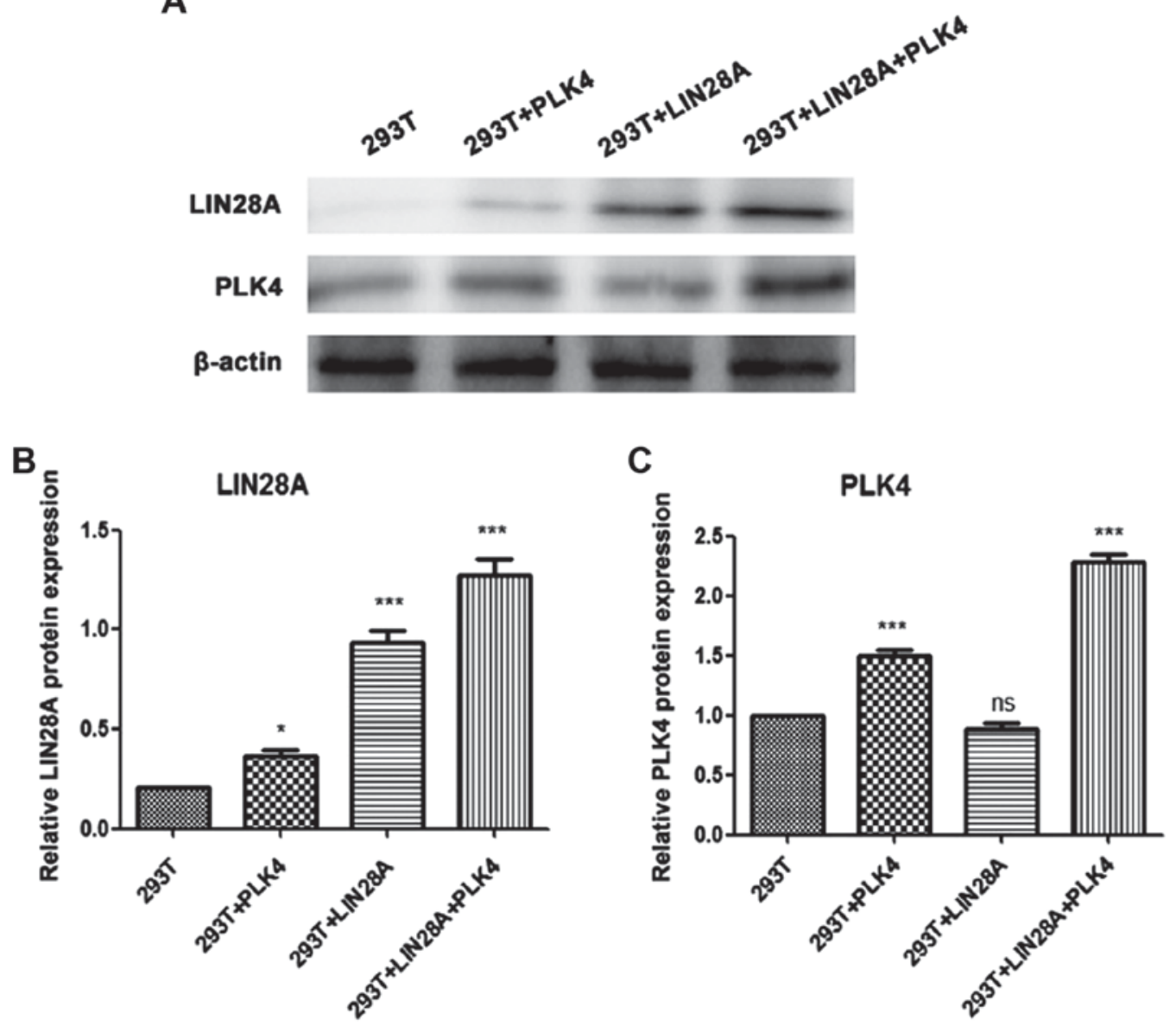

Figure 5. The levels of LIN28A and PLK4 in 293T cell lines transfected with LIN28A, PLK4 or LIN28A + PLK4 vectors, respectively. (A) Western blotting of LIN28A and PLK4 in 293T cell lines transfected with LIN28A, PLK4 or LIN28A + PLK4 vectors, respectively. Quantitative analyses of each gray numerical value of (B) LIN28A and (C) PLK4 vs. $\beta$-actin. Data were presented as the mean \pm standard deviation. "P<0.05; ${ }^{* * *} \mathrm{P}<0.001$ vs. $293 \mathrm{~T}$ cells. PLK4, Polo-like kinase 4; LIN28A, LIN28 homolog A.

expression in SKOV3, 3AO and OVCAR-3 cell lines exhibited no statistical significance. The expression level of PLK4 was significantly increased in the A2780 cell line compared with the HOSEPIC $(\mathrm{P}<0.001)$. Also, the expression of PLK4 in SKOV3 and HO8910 cell lines was significantly increased compared with HOSEPIC $(\mathrm{P}<0.05)$. No significant difference was observed in the $3 \mathrm{AO}$ and OVCAR 3 cell lines compared with HOSEPIC.

To further confirm the expression of LIN28A and PLK4 mRNA in EOC, the protein expressions of LIN28A and PLK4 was evaluated in EOC cell lines. Results demonstrated that the expression of LIN28A and PLK4 proteins in A2780 cells significantly increased in the A2780 cells $(\mathrm{P}<0.001$; Fig. 4C-E). However, the expression of LIN28A in SKOV3, $3 \mathrm{AO}$ and OVCAR-3 cell lines compared with HOSEPIC exhibited no significant difference (Fig. 4D). The expression of the PLK4 protein in A2780, HO8910 and SKOV3 was increased compared with in normal epithelial cell line (Fig. 4E). Therefore, both RT-qPCR and western blotting results indicate high expression of LIN28A and PLK4 in EOC cell lines, particularly in A2780 cells.

To investigate the association between LIN28A and PLK4, 293T cells were transfected with a LIN28A plasmid, PLK4 plasmid or co-transfected with LIN28A and PLK4 plasmids. The level of LIN28A in the PLK4 transfected group and the
PLK4 + LIN28A co-transfected group were significantly increased compared with in the control group $(\mathrm{P}<0.05$; Fig. 5). The level of PLK4 in the PLK4 + LIN28a co-transfected group was significantly increased compared with the control group $(\mathrm{P}<0.001)$; whereas, there was no significant difference in PLK4 expression between the transfected LIN28A group and the plasmid control group (Fig. 5). These results indicate that PLK4 can promote the expression of LIN28A, suggesting an association between PLK4 and LIN28A; but the specific association between them and the mechanism of interaction needs to be investigated further.

LIN28A and PLK4 are associated with cell cycle proliferation, and therefore, the proliferation of cells transfected with LIN28A and PLK4 was examined. 293T cells were transfected with the LIN28A plasmid, PLK4 plasmid or co-transfected with LIN28A and PLK4 plasmid. The cell morphology and density were evaluated under a microscope and the number of cells was counted. Transfection of plasmids demonstrated little effect on cell morphology, however, the number of cells transfected with PLK4 and co-transfected with LIN28A plus PLK4 groups were significantly increased compared with the control group ( $\mathrm{P}<0.05$; Fig. 6 ). The number of cells in the LIN28A group exhibited no difference between the transfected cells and the non-transfected cells $(\mathrm{P}>0.05)$. 
A

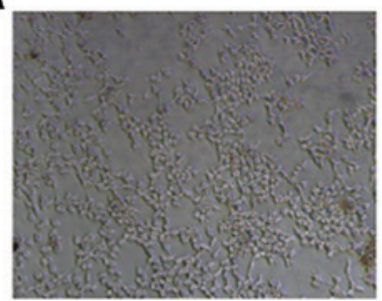

2937

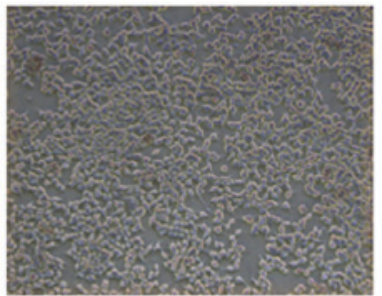

293T+PLK4

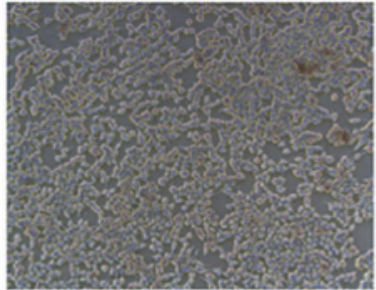

293 T+LIN28A

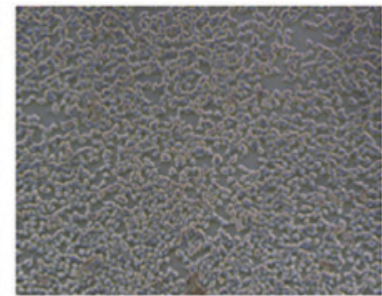

293T+LIN28A+PLK4
B

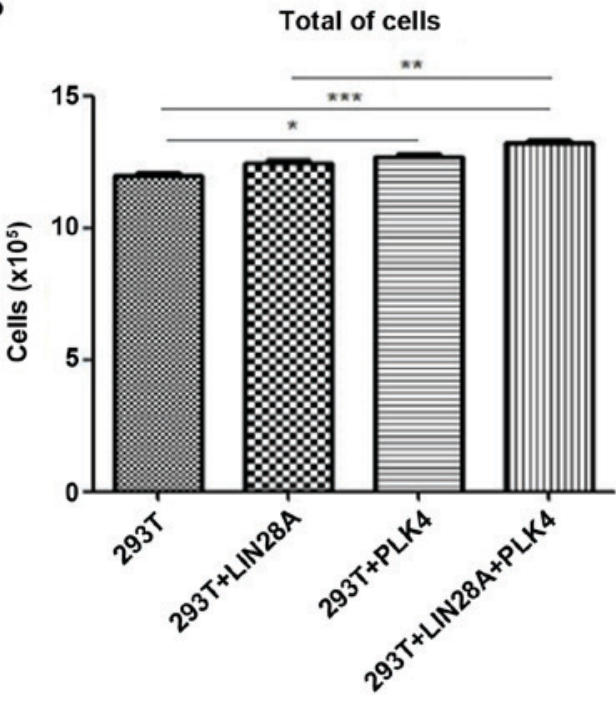

Figure 6. Proliferation of 293T cell lines transfected with LIN28A or PLK4 plasmids. (A) Morphologic observation of 293T cell lines transfected with LIN28A, PLK4 or LIN28A + PLK4 vectors, respectively. Cells were harvested following culturing for another $24 \mathrm{~h}$. Morphological observation was carried out with a Zeiss inverted microscope. (B) Total cell numbers of 293T cell lines transfected with LIN28A, PLK4 or LIN28A + PLK4 vectors, respectively. Data are presented as the mean \pm standard deviation. ${ }^{*} \mathrm{P}<0.05 ;{ }^{* *} \mathrm{P}<0.01$ and ${ }^{* * *} \mathrm{P}<0.001$. PLK4, Polo-like kinase 4; LIN28A, LIN28 homolog A.

\section{Discussion}

The role of LIN28A has been reported in a range of solid and hematological malignancies, especially in aggressive and undifferentiated tissues (9). In the present study, it was confirmed that LIN28A was highly expressed in A2780 and HO8910 cell lines. Furthermore, the expression of LIN28A was significantly increased in EOC compared with benign ovarian tissues. The results of the present study demonstrated that high LIN28A expression was a predictor of poor prognosis in EOC. It has been reported that RNA binding protein, LIN28A/LIN28B, negatively regulates let-7 through its RNA-binding domains, and loss of let-7 expression was associated with high-grade serous ovarian cancer (23). Aside from let-7, other miRNAs, including miR-26a, miR-181, miR-9, miR-30, miR-125, miR-212 and miR-27, were predicted to bind with the 3'untranslated region of LIN28 (24). Further investigation of the functional role of these miRs in ovarian cancer is required. Furthermore, exosomes secreted from the ovarian cell line, IGROV1, that has high LIN28A expression, induced 293 cell invasion and migration (25). Typically, more than one oncogenic pathway is involved in cancer progression, LIN28A contributes to the progression of breast cancer by regulating the translation of a number of oncogenes, including HER2 and HMGA1 (13). LIN28A was reported to coordinate WNT signaling to promote intestinal and colorectal adenocarcinoma invasion (26). Furthermore, high LIN28A expression was reported to be correlated with mammalian target of rapamycin (mTOR) activation by suppressing the let-7 family of miRs in an embryonal tumor with multilayered rosettes (27). Knockdown of LIN28A led to decreased mTOR activation in atypical teratoid/rhabdoid tumors (28).

In addition to regulating diverse cell functions at a transcriptional level, LIN28A binds to a consensus DNA sequence near transcription start sites and regulates associated gene expression through epigenetic DNA modification (29).
The DNA binding function of LIN28A was associated with the recruitment of 5-methylcytosine dioxygenase TET1, influencing the DNA demethylation process at gene bodies.

PLK4 is localized to a microtubule-based structure in centrosomes, called centrioles, and controls cell cycle processes, including centriole duplication and spindle assembly (30). Aberrant expression of PLK4 has been reported in a number of different cancer types of different tissues through regulating centrosome amplification, causing chromosome instability and aneuploidy $(31,32)$. Recently, Rosario et al (33) suggested that PLK4 has a role in regulating cell spreading and mobility in cancer progression. PLK4 promotes cancer invasion and metastasis involved via actin-related protein 2/3 complex-mediated cytoskeletal rearrangement (34). In the current study, the expression of PLK4 protein in A2780, HO8910 and SKOV3 cell lines was increased compared with normal epithelial cells. PLK4 expression was abnormally high in stage III/IV and could serve as a prognostic marker in EOC. The deregulation of PLK4 was shown to trigger growth interest in anti-cancer drug development (35). CFI-400945 is a potent PLK4 inhibitor that controls the number of centrosomes, and is efficient and well tolerated in animal models of breast and ovarian cancer (36). The FDA has approved the fumarate of CFI-400945 for breast cancer treatment in phase I clinical trials (35).

However, PLK4 downregulation was observed in hepatocellular carcinoma (HCC) tissues and HCC cell lines (37). The occurrence of spontaneous liver and lung tumors in PLK4 haploinsufficiency mice was $>15$ times compared with wild type mice (38). Decreased PLK4 was associated with enhanced promoter methylation of PLK4 CpG islands (39). PLK4 is susceptible to aberrant DNA methylation with age and in a number of other tumor types. However, hypermethylation of PLK4 occurred in a p53 dependent manner.

The present study demonstrated that LIN28A is positively correlated with PLK4. Both LIN28 and PLK4 promote cell 
proliferation in solid tumors. Co-transfection of cells with LIN28 and PLK4 resulted in faster growth compared with transfection with LIN28 or PLK4 in 293T cells. LIN28A and PLK4 regulate Cdc25 phosphatase, which is important for cell cycle transition and DNA damage. It is observed that Cdc25 is frequently altered in a variety of types of human cancer (40). It was demonstrated that high expression of Cdc $25 \mathrm{~A}$ and $\mathrm{Cdc} 25 \mathrm{~B}$ is associated with poor prognosis in patients with ovarian cancer, while $\mathrm{Cdc} 25 \mathrm{C}$ is undetected in the majority of patients (41). The expression of $\mathrm{Cdc} 25 \mathrm{C}$ may be regulated by other proteins. PLK4 was predicted to be the target of LIN28 as detected by RNA-protein immunoprecipitation coupled with genome-wide sequencing technique (42). Further examination is required to determine whether LIN28A interacts with PLK4 directly or indirectly.

EOC is highly heterogeneous, including several histological subtypes, and the recurrence rate for patients with advanced stages of disease is high (43). Therefore, the understanding of the molecular mechanisms of EOC is difficult, and only few biomarkers are effective for EOC. Over the past decade, carbohydrate antigen-125 (CA125) has served as a marker in first line screening for EOC. CA125 is recommended as a common marker for patients with serious EOC, but has low sensitivity and specificity in the early stage of disease. Secretory protein 4 (HE4) has been reported to have increased specificity compared with CA125 (44). The Risk of Ovarian Malignancy Algorithm, which combines HE4, CA125 and menopausal status of patients, is also utilized for the diagnosis of ovarian cancer (45). Recently, somatic or germinal mutations of BRCA1 and BRCA2 genes served as novel biomarkers in EOC with better performance to predict patient survival (46).

A number of drugs used for EOC treatment target mitotically active cells, therefore, inference with mitosis is important for anti-cancer treatment (47). High expression of PLK4 indicates poor prognosis and a PLK4 inhibitor could be a potential anti-cancer agent, which may overcome the resistance to platinum-based chemotherapy. High expression of stem factor LIN28A is correlated with advanced grade EOC and may have impact on the selection of drug-resistant phenotype due to the heterogeneity (48). The current study indicated that PLK4 and LIN28A co-expression could predict poor prognosis of EOC in stages III/IV. The most effective way to combine these biomarkers is dependent on more delicate clinical analysis and systemic models.

In conclusion, the present study demonstrated that PLK4 and LIN28A are overexpressed in ovarian cancer tissues and cell lines. High expression of LIN28A and PLK4 was correlated with high-grade pathological stage. There exists a positive association between LIN28A and PLK4. The survival period of patients with LIN28A and PLK4 double positive expression was significantly decreased compared with single positive or double negative patients $(\mathrm{P}<0.05)$. The results of the present study suggested that LIN28A and PLK4 may function as valuable prognostic biomarkers for EOC. However, the mechanism responsible for LIN28A and PLK4 interaction and its downstream effect requires further evaluation.

\section{Acknowledgements}

Not applicable.

\section{Funding}

The present study was supported by the following grants: Jiangsu Provincial Special Program of Medical Science (grant no. BL2013024), the National Natural Science Foundation of China (grant nos. 1601280020, 1601280030 and 1601270038) and the Program of Innovative Research Team of Jiangsu Province (grant nos. 201810299089Y and 201810299592W).

\section{Availability of data and materials}

The datasets used or analyzed during the current study are available from the corresponding author on reasonable request.

\section{Authors' contributions}

QS designed the experiments. YH, HW and MY performed the experiments. XY, RS, XN, XC, PY, MC, XL, GS and $\mathrm{XZ}$ analyzed the patient data. HW prepared the manuscript. QS provided suggestions for revision. All authors read and approved the final manuscript.

\section{Ethics approval and consent to participate}

The study was approved by the Ethics Committee of Jiangsu University and informed consent was obtained from all the recruited subjects.

\section{Patient consent for publication}

Informed consent was obtained from all the recruited subjects.

\section{Competing interests}

The authors declare that they have no competing interests.

\section{References}

1. Matz M, Coleman MP, Sant M, Chirlaque MD, Visser O, Gore M and Allemani C; the CONCORD Working Group: The histology of ovarian cancer: Worldwide distribution and implications for international survival comparisons (CONCORD-2). Gynecol Oncol 144: 405-413, 2017.

2. Kurman RJ and Shih Ie M: Molecular pathogenesis and extraovarian origin of epithelial ovarian cancer-shifting the paradigm. Hum Pathol 42: 918-931, 2011.

3. The trouble with ovarian cancer. Lancet 374: 1302, 2009.

4. Kurman RJ and Shih Ie M: The dualistic model of ovarian carcinogenesis: Revisited, revised, and expanded. Am J Pathol 186: 733-747, 2016.

5. Chien J, Kuang R, Landen C and Shridhar V: Platinum-sensitive recurrence in ovarian cancer: The role of tumor microenvironment. Front Oncol 3: 251, 2013.

6. Burgos-Ojeda D, Rueda BR and Buckanovich RJ: Ovarian cancer stem cell markers: Prognostic and therapeutic implications. Cancer Lett 322: 1-7, 2012

7. Carmel-Gross I, Bollag N, Armon L and Urbach A: LIN28: A stem cell factor with a key role in pediatric tumor formation. Stem Cells Dev 25: 367-377, 2016.

8. Heo I, Joo C, Cho J, Ha M, Han J and Kim VN: Lin28 mediates the terminal uridylation of let-7 precursor MicroRNA. Mol Cell 32: 276-284, 2008.

9. Zhou J, Ng SB and Chng WJ: LIN28/LIN28B: An emerging oncogenic driver in cancer stem cells. Int J Biochem Cell Biol 45: 973-978, 2013. 
10. Shyh-Chang N and Daley GQ: Lin28: Primal regulator of growth and metabolism in stem cells. Cell Stem Cell 12: 395-406, 2013.

11. Feng C, Neumeister V, Ma W, Xu J, Lu L, Bordeaux J, Maihle NJ, Rimm DL and Huang Y: Lin28 regulates HER2 and promotes malignancy through multiple mechanisms. Cell Cycle 11: 2486-2494, 2012.

12. Ma W, Ma J, Xu J, Qiao C, Branscum A, Cardenas A, Baron AT, Schwartz P, Maihle NJ and Huang Y: Lin28 regulates BMP4 and functions with Oct4 to affect ovarian tumor microenvironment. Cell Cycle 12: 88-97, 2013.

13. Li N, Zhong X, Lin X, Guo J, Zou L, Tanyi JL, Shao Z, Liang S, Wang LP, Hwang WT, et al: Lin-28 homologue A (LIN28A) promotes cell cycle progression via regulation of cyclin-dependent kinase 2 (CDK2), cyclin D1 (CCND1), and cell division cycle 25 homolog A (CDC25A) expression in cancer. J Biol Chem 287: 17386-17397, 2012.

14. de Cárcer G, Manning G and Malumbres M: From Plk1 to Plk5: Functional evolution of polo-like kinases. Cell Cycle 10: 2255-2262, 2011

15. Takai N, Hamanaka R, Yoshimatsu J and Miyakawa I: Polo-like kinases (Plks) and cancer. Oncogene 24: 287-291, 2005.

16. Weiss L and Efferth T: Polo-like kinase 1 as target for cancer therapy. Exp Hematol Oncol 1: 38, 2012.

17. Lohse I, Mason J, Cao PM, Pintilie M, Bray M and Hedley DW: Activity of the novel polo-like kinase 4 inhibitor CFI-400945 in pancreatic cancer patient-derived xenografts. Oncotarget 8 : 3064-3071, 2017.

18. Denu RA, Zasadil LM, Kanugh C, Laffin J, Weaver BA and Burkard ME: Centrosome amplification induces high grade features and is prognostic of worse outcomes in breast cancer. BMC Cancer 16: 47, 2016.

19. Li Z, Dai K, Wang C, Song Y, Gu F, Liu F and Fu L: Expression of polo-like kinase 4(PLK4) in breast cancer and its response to taxane-based neoadjuvant chemotherapy. J Cancer 7: 1125-1132, 2016.

20. Bonni S, Ganuelas ML, Petrinac S and Hudson JW: Human Plk4 phosphorylates Cdc25C. Cell Cycle 7: 545-547, 2008

21. Mutch DG and Prat J: 2014 FIGO staging for ovarian, fallopian, tube and peritoneal cancer. Gynecol Oncol 133: 401-404, 2014

22. Livak KJ and Schmittgen TD: Analysis of relative gene expression and the 2(-Delta Delta C(T)) method. Methods 25: 402-408, 2001.

23. Helland $\AA$, Anglesio MS, George J, Cowin PA, Johnstone CN, House CM, Sheppard KE, Etemadmoghadam D, Melnyk N, Rustgi AK, et al: Deregulation of MYCN, LIN28B and LET7 in a molecular subtype of aggressive high-grade serous ovarian cancers. PLoS One 6: e18064, 2011.

24. Wang T, Wang G, Hao D, Liu X, Wang D, Ning N and Li X: Aberrant regulation of the LIN28A/LIN28B and let-7 loop in human malignant tumors and its effects on the hallmarks of cancer. Mol Cancer 14: 125, 2015.

25. Enriquez VA, Cleys ER, Da Silveira JC, Spillman MA, Winger QA and Bouma GJ: High LIN28A expressing ovarian cancer cells secrete exosomes that induce invasion and migration in HEK293 cells. Biomed Res Int 2015: 701390, 2015.

26. Tu HC, Schwitalla S, Qian Z, LaPier GS, Yermalovich A, Ku YC, Chen SC, Viswanathan SR, Zhu H, Nishihara R, et al: LIN28 cooperates with WNT signaling to drive invasive intestinal and colorectal adenocarcinoma in mice and humans. Genes Dev 29: 1074-1086, 2015.

27. Spence T, Perotti C, Sin-Chan P, Picard D, Wu W, Singh A, Anderson C, Blough MD, Cairncross JG, Lafay-Cousin L, et al: A novel C19MC amplified cell line links Lin28/let-7 to mTOR signaling in embryonal tumor with multilayered rosettes. Neuro Oncol 16: 62-71, 2014

28. Rubens JA, Wang SZ, Price A, Weingart MF, Allen SJ, Orr BA, Eberhart CG and Raabe EH: The TORC1/2 inhibitor TAK228 sensitizes atypical teratoid rhabdoid tumors to cisplatin-induced cytotoxicity. Neuro Oncol 19: 1361-1371, 2017.

29. Zeng Y, Yao B, Shin J, Lin L, Kim N, Song Q, Liu S, Su Y, Guo JU, Huang L, et al: Lin28A binds active promoters and recruits Tet1 to regulate gene expression. Mol Cell 61: 153-160, 2016.
30. Sillibourne JE and Bornens M: Polo-like kinase 4: The odd one out of the family. Cell Div 5: 25, 2010.

31. Shinmura K, Kurabe N, Goto M, Yamada H, Natsume H, Konno $\mathrm{H}$ and Sugimura H: PLK4 overexpression and its effect on centrosome regulation and chromosome stability in human gastric cancer. Mol Biol Rep 41: 6635-6644, 2014

32. Ling H, Hanashiro K, Luong TH, Benavides L and Fukasawa K: Functional relationship among PLK2, PLK4 and ROCK2 to induce centrosome amplification. Cell Cycle 14: 544-553, 2015.

33. Rosario CO, Kazazian K, Zih FS, Brashavitskaya O, Haffani Y, Xu RS, George A, Dennis JW and Swallow CJ: A novel role for Plk4 in regulating cell spreading and motility. Oncogene 34: 3441-3451, 2015

34. Kazazian K, Go C, Wu H, Brashavitskaya O, Xu R, Dennis JW, Gingras AC and Swallow CJ: Plk4 promotes cancer invasion and metastasis through Arp2/3 complex regulation of the actin cytoskeleton. Cancer Res 77: 434-447, 2017.

35. Mason JM, Lin DC, Wei X, Che Y, Yao Y, Kiarash R, Cescon DW, Fletcher GC, Awrey DE, Bray MR, et al: Functional characterization of CFI-400945, a Polo-like kinase 4 inhibitor, as a potential anticancer agent. Cancer Cell 26: 163-176, 2014.

36. Yu B, Yu Z, Qi PP, Yu DQ and Liu HM: Discovery of orally active anticancer candidate CFI-400945 derived from biologically promising spirooxindoles: Success and challenges. Eur J Med Chem 95: 35-40, 2015.

37. Liu L,Zhang CZ,Cai M,Fu J, Chen GG and Yun J: Downregulation of polo-like kinase 4 in hepatocellular carcinoma associates with poor prognosis. PLoS One 7: e41293, 2012.

38. Ko MA, Rosario CO, Hudson JW, Kulkarni S, Pollett A, Dennis JW and Swallow CJ: Plk4 haploinsufficiency causes mitotic infidelity and carcinogenesis. Nat Genet 37: 883-888, 2005.

39. Ward A, Morettin A, Shum D and Hudson JW: Aberrant methylation of Polo-like kinase $\mathrm{CpG}$ islands in Plk4 heterozygous mice. BMC Cancer 11: 71, 2011.

40. Boutros R, Lobjois V and Ducommun B: CDC25 phosphatases in cancer cells: Key players? Good targets? Nat Rev Cancer 7: 495-507, 2007.

41. Broggini M, Buraggi G, Brenna A, Riva L, Codegoni AM, Torri V, Lissoni AA, Mangioni C and D'Incalci M: Cell cycle-related phosphatases CDC25A and B expression correlates with survival in ovarian cancer patients. Anticancer Res 20: 4835-4840, 2000.

42. Yang J, Bennett BD, Luo S, Inoue K, Grimm SA, Schroth GP, Bushel PR, Kinyamu HK and Archer TK: LIN28A modulates splicing and gene expression programs in breast cancer cells. Mol Cell Biol 35: 3225-3243, 2015.

43. Ueland FR: A perspective on ovarian cancer biomarkers: Past, present and yet-to-come. Diagnostics (Basel) 7: E14, 2017.

44. Wei SU, Li H and Zhang B: The diagnostic value of serum HE4 and CA-125 and ROMA index in ovarian cancer. Biomed Rep 5: 41-44, 2016.

45. Sölétormos G, Duffy MJ, Othman Abu Hassan S, Verheijen RH, Tholander B, Bast RC Jr, Gaarenstroom KN, Sturgeon CM, Bonfrer JM, Petersen PH, et al: Clinical use of cancer biomarkers in epithelial ovarian cancer: Updated guidelines from the european group on tumor markers. Int J Gynecol Cancer 26: 43-51, 2016.

46. Arts-De Jong M, De Bock GH, Van Asperen CJ, Mourits MJ, de Hullu JA and Kets CM: Germline BRCA1/2 mutation testing is indicated in every patient with epithelial ovarian cancer: A systematic review. Eur J Cancer 61: 137-145, 2016.

47. Pujade-Lauraine E, Selle F, Weber B, Ray-Coquard IL, Vergote I, Sufliarsky J, Del Campo JM, Lortholary A, Lesoin A, Follana P, et al: Volasertib versus chemotherapy in platinum-resistant or -refractory ovarian cancer: A randomized phase II groupe des investigateurs nationaux pour l'etude des cancers de l'ovaire study. J Clin Oncol 34: 706-713, 2016.

48. Peng S, Maihle NJ and Huang Y: Pluripotency factors Lin28 and Oct4 identify a sub-population of stem cell-like cells in ovarian cancer. Oncogene 29: 2153-2159, 2010.

This work is licensed under a Creative Commons Attribution-NonCommercial-NoDerivatives 4.0 International (CC BY-NC-ND 4.0) License. 